\title{
Stellar population gradients in bulges along the Hubble sequence ${ }^{\star \star \star}$
}

\section{The data}

\author{
J. Gorgas ${ }^{1}$, P. Jablonka ${ }^{2}$, and P. Goudfrooij ${ }^{3}$ \\ 1 Dpto. de Astrofísica, Facultad de Físicas, Universidad Complutense de Madrid, 28040 Madrid, Spain \\ 2 Observatoire de l'Université de Genève, Laboratoire d'Astrophysique de l'École Polytechnique Fédérale de Lausanne (EPFL), \\ 1290 Sauverny, Switzerland \\ e-mail: pascale.jablonka@obs.unige.ch \\ 3 Space Telescope Science Institute, 3700 San Martin Drive, Baltimore, MD 21218, USA
}

Received 3 November 2006 / Accepted 28 June 2007

\section{ABSTRACT}

\begin{abstract}
This is the first paper presenting our long-term project aimed at studying the nature of bulges through analyzing their stellar population gradients. We present deep spectroscopic observations along the minor axis and the data reduction for a sample of 32 bulges of edge-on spiral galaxies. We explain in detail our procedures for measuring their dynamical parameters (rotation curves and velocity dispersion profiles) and line-strength indices, including the conversion to the Lick/IDS system. Tables giving the values of the dynamical parameters and line-strength indices at each galactocentric radius are presented (in electronic form) for each galaxy of the sample. The derived line-strength gradients from this dataset will be analyzed in a forthcoming paper to set constraints on the different scenarios for the formation of the bulges.
\end{abstract}

Key words. galaxies: bulges - galaxies: stellar content - methods: data analysis - galaxies: abundances - galaxies: evolution galaxies: formation.

\section{Introduction}

Bulges of spiral galaxies are cornerstones for constraining theories of galaxy formation. Located at the centers of spiral galaxies, they hold the signature for the sequence of formation of the different sub-systems building a spiral galaxy: halo, disk, and bulge. While the mass of disks of spiral galaxies in the local universe is approximately constant among spirals of different Hubble types (e.g., Arimoto \& Jablonka 1991), the prominence of bulges varies widely along the Hubble sequence. Hence, bulges are likely to hold important clues to our understanding of galaxy evolution and the nature of the Hubble sequence.

Formation scenarios for bulges can be divided into two categories. One of them states that bulges are formed in a similar way to low- to intermediate-mass elliptical galaxies, based on the strong similarities between the global properties of many bulges and of elliptical galaxies. Bulges and (small) ellipticals populate the same location in the fundamental plane (Bender et al. 1992; Falcón-Barroso et al. 2002), and they form a continuous sequence in the $V_{\max } / \sigma$ vs. ellipticity diagram (being supported by rotation; Bender et al. 1992). Furthermore, spectroscopic studies of the central part of bulges have shown that their mass-metallicity relation (when derived from $\alpha$ elements, e.g. the $\mathrm{Mg}_{2}$ index) is consistent with the relation found for

* Based on observations collected at the Roque de los Muchachos Observatory and at the European Southern Observatory, proposal numbers 58.A-0192(A), 59.A-0774(A) and 61.A-0326(A).

$\star \star 2$ datasets are only available in electronic form at the CDS via anonymous ftp to cdsarc.u-strasbg. fr $(130.79 .128 .5)$ or via http://cdsweb.u-strasbg.fr/cgi-bin/qcat?J/A+A/474/1081 elliptical galaxies (Jablonka et al. 1996; Idiart et al. 1996; Moorthy \& Holtzman 2006), but see also Prugniel, Maubon, \& Simien (2001) and Proctor \& Sansom (2002). We refer the reader to the introduction in Jablonka et al. (2007, hereafter Paper II) for a discussion of previous spectroscopic studies of bulges.

The other popular type of formation scenario for bulges is the "secular evolution" scenario in which bulges form from disk material through redistribution of angular momentum. This scenario involves stellar bars driving gas from the disk into the central region of the galaxy, thus triggering star formation (e.g., Pfenniger \& Norman 1990; Norman et al. 1996; Kormendy \& Kennicutt 2004). If enough mass is accreted, the bar itself will dissolve, and the orbits of stars involved in the process will yield a bulge-like spatial distribution. Galaxies would thus evolve from late to earlier types along the Hubble sequence. Observational support for secular evolution has been provided mainly for late-type spiral galaxies ( $\sim \mathrm{Sb}$ and later), including correlations between the scale lengths of bulges and disks (Courteau et al. 1996; McArthur et al. 2003) and the radial light distribution of bulges of late-type spirals being closer to exponential than to classical de Vaucouleurs $R^{1 / 4}$ profiles (Balcells et al. 2003; de Jong et al. 2004).

Stellar population studies should be able to place important constraints on the formation mechanisms for bulges. If bulges form mainly through dissipative collapse without significant subsequent merging, simulations indicate that the slope of radial metallicity gradients should steepen with increasing galaxy mass and luminosity (Carlberg 1984). Simulations of dissipative merging of gas-rich disks has also been shown to produce 
metallicity gradients that steepen with increasing mass (e.g., Bekki \& Shioya 1999). Conversely, the impact of secular evolution on population gradients is expected to be rather different. Simulations including gas and effects of star formation show that disk gas fueled to the central regions renders a central region that is younger and more metal rich than the outer regions, whereas metallicity gradients outside the central region flatten out significantly $\sim 1$ Gyr after formation of a bar, both for gas and stars (Friedli et al. 1994; Friedli 1998). Observational evidence for this effect exists, albeit so far only for the gas component in spiral disks. Several studies have shown that global radial gradients of the gas metallicity in barred spirals are shallower than gradients in unbarred spirals of the same Hubble type (Vila-Costas \& Edmunds 1992; Zaritsky et al. 1994; Martin \& Roy 1994).

In terms of stellar population gradients in bulges, past studies have mainly used broad-band imaging. The studies of Balcells \& Peletier (1994) and Peletier et al. (1999) used optical and near-infrared colors and found that color gradients of luminous bulges increase with luminosity, whereas faint bulges show stronger gradients than expected from the trend observed for the luminous bulges. However, information in colors is generally rather degenerate in age, metallicity, and/or extinction.

Spectroscopic studies allow the measurement of line strength indices that are insensitive to dust extinction and allow a cleaner separation of age and metallicity of a stellar population. While line strength gradients have been measured for many elliptical and S0 galaxies using the Lick/IDS system of indices (e.g., Carollo et al. 1993; González \& Gorgas 1996; Fisher et al. 1996; Mehlert et al. 2003; Kuntschner et al. 2006; Sánchez-Blázquez et al. 2006a), such data are relatively hard to acquire for bulges of spirals at high enough signal-to-noise ratios, given the often low surface brightness of bulges and the difficulty of disentangling the observed spectra into bulge and disk components. This is especially difficult for late-type spirals in face-on or moderately inclined configurations.

With this in mind, we embarked on an extensive spectroscopic survey of 32 spiral galaxies in an edge-on configuration to avoid contamination by disk light and with Hubble types ranging from S0 to Sc. Early results from subsets of the data acquired during this survey were published in Goudfrooij et al. (1999), Jablonka et al. (2002), and Gorgas et al. (2003). In this first paper on the final and comprehensive results of this survey, we present the extensive data reduction and analysis procedures leading to the final line strength measurements. The galaxy sample is presented in Sect. 2. Section 3 describes the observations, and Sect. 4 provides details on the data reduction procedures. Section 5 contains a description of the determination of dynamical parameters (radial velocities and velocity dispersions as a function of bulge radius), and the line strength index measurements themselves are described in Sect. 6. Finally, Sect. 7 contains a summary of this work.

\section{The sample}

We selected a sample of 32 genuine (or close to) edge-on spiral galaxies. Galaxies in the northern hemisphere were selected from the Uppsala General Catalogue (Nilson 1973), while southern galaxies were selected from the ESO/Uppsala catalogue (Lauberts 1982). As shown in Fig. 1, the galaxies span a wide range in Hubble types (from $\mathrm{S} 0$ to $\mathrm{Sc}$ ). Given the edge-on configuration of the galaxies, precise morphological classification is difficult. Due to this we considered a Hubble T-type uncertainty of \pm 1 , which is also a fair representation of the catalogue-tocatalogue variations for a given galaxy.

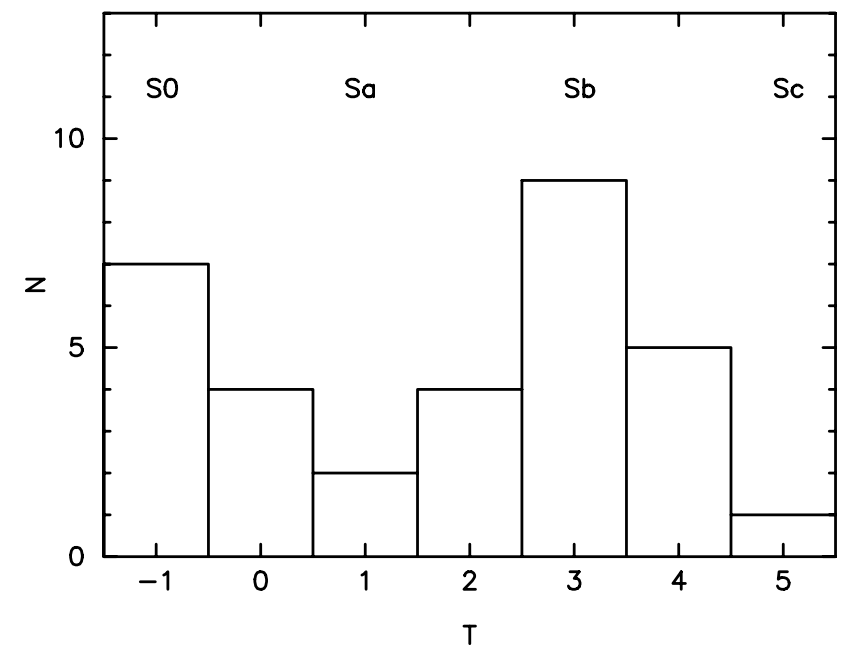

Fig. 1. Histogram showing the distribution in Hubble types of the galaxy sample.

All the galaxies are nearby objects, with radial velocities between 550 and $6200 \mathrm{~km} \mathrm{~s}^{-1}$, and were chosen to cover a range in bulge luminosity $\left(-17.5>M_{\mathrm{V}}>-20.5\right)$. Table 1 lists the galaxy sample and some relevant parameters, including the corresponding bulge types (from Lütticke et al. 2000) and the local environment, which also varies significantly among the sample galaxies. A few galaxies have previous photometric and spectroscopic observations from other authors (see Sect. 6) and were included for comparison purposes.

\section{Spectroscopic observations}

Spectroscopic observations of the galaxy sample were obtained during five observing runs at three different telescopes: The northern galaxies were observed at the 2.5-m Isaac Newton Telescope (INT) of the Isaac Newton Group of telescopes on the island of La Palma (Spain). For the southern galaxies, we used the 3.6-m ESO telescope and 3.6-m New Technology Telescope (NTT), both at ESO La Silla observatory (Chile). The main parameters of the instrument configurations and other details of the observing runs are listed in Table 2. Using slit widths from 1.0 to 2.0 arcsec, the different spectrographs allowed us to obtain spectra at a resolution of typically $4 \AA$ ( $F W H M$, as measured from the widths of arc lines), except for run 3 (the ESO 3.6-m run), where a resolution of $8.3 \AA$ was attained. The spectral range from around $\lambda 3900 \AA$ to $\lambda 5500 \AA$ (with the exception of the ESO NTT runs, in which the spectra extended redward to $\lambda 6650 \AA$ ), was chosen to include the main prominent spectral features of the blue-yellow spectra of the galaxies, in particular most of the Lick/IDS line-strength indices.

Table 1 includes some relevant observational parameters for the galaxy sample. The spectrograph slit was oriented along the minor axis of the bulges. Thus, effects of contamination by disk light are only expected for the very central regions of the sample galaxies. Exposure times ranged from 1.5 to $5 \mathrm{~h}$ per galaxy, providing high signal-to-noise $(S / N)$ spectra out to the outer regions of most bulges. We obtained reliable spectra out to the effective radius $\left(r_{\text {eff }}\right)$ for every galaxy in our sample. Furthermore, our spectra reached $S / N>10$ (per $\AA$ and per arcsec in the spatial direction) beyond $2 r_{\mathrm{eff}}$ for $80 \%$ of the sample galaxies. Some observations of nearby bulges were especially deep, providing useful spectra out to several kpc from the centers of the bulges 
Table 1. The galaxy sample.

\begin{tabular}{|c|c|c|c|c|c|c|c|c|c|c|c|c|}
\hline & Type & $T$ & $B_{\mathrm{T}}$ & $i$ & Bulge type $^{a}$ & Environment & $\operatorname{Run}^{b}$ & $\mathrm{PA}^{c}$ & Exp. $^{d}$ & $r_{\mathrm{eff}}^{e}$ & $\Delta r_{\mathrm{eff}}$ & $V_{\max }^{f}$ \\
\hline NGC 522 & Sc & 4.1 & 13.98 & 90 & box & & 2 & 123 & 8200 & 3.5 & 0.7 & 196.1 \\
\hline NGC 585 & $\mathrm{Sa}$ & 1.0 & 14.06 & 90 & close to box & & 4,5 & 86 & 7200 & 2.4 & 0.7 & 246.6 \\
\hline NGC 678 & $\mathrm{SBb}$ & 3.0 & 13.31 & 90 & box & cluster & 1 & 168 & 12000 & 23.3 & 1.4 & 169.0 \\
\hline NGC 891 & $\mathrm{SAb}$ & 3.0 & 10.83 & 88 & box & & 1 & 112 & 10500 & 13.2 & 0.7 & 212.0 \\
\hline NGC 973 & Sbc & 3.2 & 14.17 & 90 & box & & 2 & 138 & 13800 & 3.4 & 0.4 & 270.0 \\
\hline NGC 1032 & S0-a & 0.4 & 12.74 & 90 & elliptical & companions? & 1 & 158 & 9000 & 11.4 & 1.1 & 283.9 \\
\hline NGC 1184 & S0-a & 0.0 & 12.95 & 90 & close to box & & 2 & 79 & 8200 & 11.3 & 1.9 & \\
\hline NGC 1351A & SBbc & 4.5 & 14.16 & 80 & elliptical & cluster & 5 & 132 & 5400 & 5.8 & 0.8 & 90.7 \\
\hline NGC 1886 & Sab & 3.9 & 13.80 & 90 & peanut & isolated & 3 & 60 & 10800 & 1.6 & 0.3 & 154.6 \\
\hline NGC 3957 & SA0 & -1.0 & 13.03 & 90 & close to box & cluster & 3 & 173 & 7200 & 4.0 & 0.8 & \\
\hline NGC 5084 & S0 & -1.8 & 11.55 & 90 & elliptical & cluster & 3 & 80 & 7200 & 38.1 & 11.6 & 310.3 \\
\hline NGC 6010 & S0-a & 0.4 & 12.95 & 90 & & & 1 & 15 & 13800 & 4.2 & 0.9 & 148.8 \\
\hline NGC 6829 & $\mathrm{Sb}$ & 3.0 & 15.09 & 90 & & & 2 & 121 & 12800 & 7.1 & 1.4 & 211.9 \\
\hline NGC 7183 & S0 & -0.7 & 13.14 & 90 & close to box & & 5 & 77 & 7200 & 2.5 & 0.5 & 209.9 \\
\hline NGC 7264 & $\mathrm{Sb}$ & 3.1 & 14.58 & 90 & elliptical & & 2 & 147 & 13000 & 1.2 & 0.4 & 248.0 \\
\hline NGC 7332 & So & -2.0 & 12.56 & 90 & close to box & & 1 & 65 & 15000 & 7.5 & 1.1 & 121.3 \\
\hline NGC 7396 & $\mathrm{Sa}$ & 1.0 & 13.85 & 60 & elliptical & & 2 & 13 & 13000 & 25.2 & 1.6 & 360.7 \\
\hline NGC 7703 & S0 & -2.0 & 14.41 & 90 & elliptical & cluster & 2 & 57 & 6400 & 4.8 & 2.0 & 159.5 \\
\hline NGC 7814 & SAab & 2.0 & 11.68 & 67 & elliptical & & 1 & 45 & 18400 & 33.5 & 2.0 & 231.0 \\
\hline IC 1711 & $\mathrm{Sb}$ & 3.0 & 14.53 & 90 & elliptical & & 2 & 133 & 6200 & 2.1 & 0.5 & 173.4 \\
\hline IC 1970 & SAb & 3.1 & 13.36 & 88 & elliptical & & 4 & 75 & 7200 & 8.1 & 1.0 & 118.9 \\
\hline IC 2531 & $\mathrm{Sb}$ & 4.9 & 12.88 & 90 & peanut & cluster & 3 & 75 & 7200 & 2.3 & 0.3 & 228.1 \\
\hline IC 5176 & $\mathrm{SABbc}$ & 4.4 & 13.02 & 90 & elliptical & companions? & 5 & 151 & 7200 & 4.6 & 0.3 & 164.2 \\
\hline IC 5264 & Sab & 2.3 & 13.58 & 90 & elliptical & group & 4,5 & 82 & 12600 & 2.8 & 0.1 & 230.6 \\
\hline UGC 10043 & $\mathrm{Sbc}$ & 4.1 & 15.17 & 90 & & cluster & 2 & 61 & 12200 & 1.0 & 0.3 & 143.6 \\
\hline UGC 11552 & Sab & 2.0 & 15.68 & 75 & & & 5 & 16 & 7200 & 11.7 & 0.6 & 243.0 \\
\hline UGC 11587 & So & -1.9 & 14.52 & 90 & & & 1 & 76 & 15000 & 5.7 & 0.7 & \\
\hline ESO 079-003 & $\mathrm{SBb}$ & 3.1 & 13.91 & 90 & close to box & & 4,5 & 134 & 10800 & 5.9 & 2.0 & 192.2 \\
\hline ESO 234-053 & So & -1.9 & 14.32 & 90 & & & 5 & 81 & 5400 & 1.7 & 0.1 & \\
\hline ESO 311-012 & S0-a & 0.1 & 12.38 & 90 & box & isolated & 3 & 14 & 7200 & 7.4 & 1.3 & 95.9 \\
\hline ESO 443-042 & $\mathrm{Sb}$ & 3.1 & 13.85 & 90 & peanut & companions? & 3 & 130 & 10800 & 3.8 & 0.7 & 166.3 \\
\hline ESO 512-012 & Sbc & 3.2 & 14.42 & 90 & peanut & & 3 & 113 & 5400 & 1.4 & 0.3 & 224.9 \\
\hline
\end{tabular}

${ }^{a}$ From Lütticke et al. (2000). ${ }^{b}$ Run number in which each galaxy was observed. ${ }^{c}$ Position angle of the long slit. ${ }^{d}$ Total exposure time (in seconds). ${ }^{e}$ Effective radius (in arcseconds) of the bulge, computed as explained in Sect. $4 .{ }^{f}$ Maximum rotational velocity (in $\mathrm{km} \mathrm{s}^{-1}$ ) along the disk major axis, corrected from inclination when necessary. Hubble type $T$, total apparent magnitude $B_{\mathrm{T}}$, galaxy inclination $i$ and $V_{\max }$ have been taken from HyperLeda (http://leda.univ-lyon1.fr/).

(e.g., $\sim 7 \mathrm{kpc}$ for NGC 5084 and $\sim 4 \mathrm{kpc}$ for NGC 7814). In the central regions we obtained spectra with $\mathrm{S} / \mathrm{N}$ ranging from 20 to 240 , depending, among other factors, on the obscuration by dust lanes within the galaxy disk.

In each observing run we also acquired spectra of a number of template stars from the Lick/IDS library (Worthey et al. 1994) for calibration purposes (see below), as well as several spectrophotometric standards to calibrate the spectra in flux.

\section{Data reduction}

The reduction of the data was performed with the $\mathrm{RED}_{\mathrm{m}}^{\mathrm{uc}} \mathrm{E}^{1}$ package (Cardiel 1999). We carried out a standard reduction procedure for spectroscopic data: bias and dark subtraction, cosmic ray cleaning, flat-fielding (using observations of tungsten lamps and twilight skies to correct from high and low frequency variations respectively), C-distortion correction, wavelength calibration, S-distortion correction and recentering of the spectra, sky subtraction, atmospheric and interstellar extinction correction, relative flux calibration, and spectrum extraction. $\mathrm{RED}_{\mathrm{m}}^{\mathrm{uC}} \mathrm{E}$ is a package specifically written to reduce long-slit spectroscopic observation. Its main advantage is that, for each observed data

\footnotetext{
${ }^{1}$ http://www.ucm.es/info/Astrof/software/reduceme/ reduceme.html
}

frame (including calibration ones), it creates an associated error frame at the beginning of the reduction procedure. From this point, error and data frames are processed in parallel, translating all the uncertainties in the manipulation of the data into the error frames, hence providing very accurate estimates of the random errors associated with the final spectra (see Cardiel et al. 1998).

Although most of the reduction steps were performed using standard procedures for long-slit reduction, we give below some comments on steps of particular importance:

\section{(i) Wavelength calibration}

Spectra were converted to a linear wavelength scale using about 90 arc lines fitted by 5 th to 7 th-order polynomials. The rms dispersion of the fitted position of arc lines was typically $0.25-0.30 \AA$.

\section{(ii) Spectral rectification and centering}

As usual with CCD spectrographs, the galaxy spectra were not perfectly aligned with the detector rows. To correct for this effect (which is crucial when measuring line-strength gradients), we fitted a Cauchy function to the number of counts in a spatial interval around the location of maximum counts for each spectral resolution element, hence determining the position of the galaxy center as a function of wavelength. In galaxies with dust lanes obscuring the light of the central region of the bulge, these central parts were masked when fitting the luminosity profiles. Experiments with galaxies without dust lanes showed that we 
Table 2. Instrumental details of the observing runs.

\begin{tabular}{cccccccc}
\hline \hline Run & Dates & Telescope & Instrument & $\begin{array}{c}\text { Dispersion } \\
\AA / \text { pixel }\end{array}$ & $\begin{array}{c}\text { Spectral range } \\
\AA\end{array}$ & $\begin{array}{c}\text { Slit width } \\
\text { arcsec }\end{array}$ & $\begin{array}{c}F W H M^{a} \\
\AA\end{array}$ \\
\hline 1 & $13 / 08 / 96-16 / 08 / 96$ & INT 2.5 m & IDS & 1.6 & $3908-5535$ & 2.0 & 3.9 \\
2 & $31 / 08 / 97-03 / 09 / 97$ & INT 2.5 m & IDS & 1.6 & $3908-5535$ & 2.0 & 4.3 \\
3 & $08 / 03 / 97-11 / 03 / 97$ & ESO 3.6 m & EFOSC & 3.4 & $3776-5504$ & 1.5 & 8.3 \\
4 & $23 / 09 / 97-25 / 09 / 97$ & NTT 3.6 m & EMMI & 1.3 & $3971-6649$ & 1.0 & 3.7 \\
5 & $23 / 08 / 98-26 / 08 / 98$ & NTT 3.6 m & EMMI & 1.3 & $3985-6649$ & 1.5 & 4.9 \\
\hline
\end{tabular}

${ }^{a}$ Spectral resolution as measured from arc lines.

could still trace the position of the galaxy center. The resulting map was then fitted by a low-order polynomial, which was in turn used to straighten the spectra. As a result of this process, the central spectrum of the galaxy is moved to the center of a detector row so that symmetric spectra on each side of that row correspond to the same galactocentric radius.

(iii) Sky subtraction

Accurate sky subtraction is critical for studies like this, since we aim to analyze spectra at light levels corresponding to only a few per cent of the sky signal. We refer the reader to Cardiel et al. (1995) for a description of the sky subtraction effects on the measurements of line-strength indices. For each of our galaxy observations we generated a sky image by fitting, for each pixel in the wavelength direction, a low-order polynomial using the regions close to the slit ends. In most cases, the galaxies fill only a small region of the slit, so this synthetic sky image is free of contamination from the galaxy light. However, this is not the case for some of the larger galaxies of the sample (like NGC 7814 and NGC 5084). In these cases, we fitted de Vaucouleurs' $r^{1 / 4}$ profiles to the surface brightness distribution of the galaxies along the slit, estimating the relative contribution of the galaxy to the regions from where the sky was extracted. We then "decontaminated" the sky frames by subtracting scaled and averaged galaxy spectra. To assess the quality of the sky subtraction, we looked for residuals due to sky emission lines, making sure that they were completely removed, even in the outer parts of the more extended galaxies.

(iv) Measurement of effective radii

Effective radii for all the bulges of the sample were measured by fitting de Vaucouleurs' profiles to the radial surface brightness profiles determined by collapsing the spectra in the wavelength direction. The central regions, affected in many cases by dust lanes, were removed from the fit. In any case, we never used spectra at radii typically below 2 arcsec. On the other hand, the fits were quite insensitive to the outer radius cutoff, although deviations of the luminosity profiles from a straight line in the logarithmic plots were sometimes observed. The derived effective radii are listed in Table 1 . The associated errors, in the last column, reflect the sensitivity of the derived radii to the particular choice of the region to be fitted. Note that we are not assuming that the bulges do follow a de Vaucouleurs luminosity profile. (A Sersic profile with an exponent $n$ closer to 1 provides a better fit in many cases, see Andreakis et al. 1995.) However, the derived effective radii provide us with a well-defined parameter to express the line-strength gradients (Paper II) on a homogeneous spatial scale.

\section{(v) Flux calibration}

Relative flux calibration of the spectra was achieved using exposures of spectrophotometric standards from Massey et al. (1988), Oke (1990), and Hamuy et al. (1994). In each run we took several repeated observations of $4,5,4,3$, and 3 stars (for runs 1 to 5 respectively). In some cases we obtained exposures at

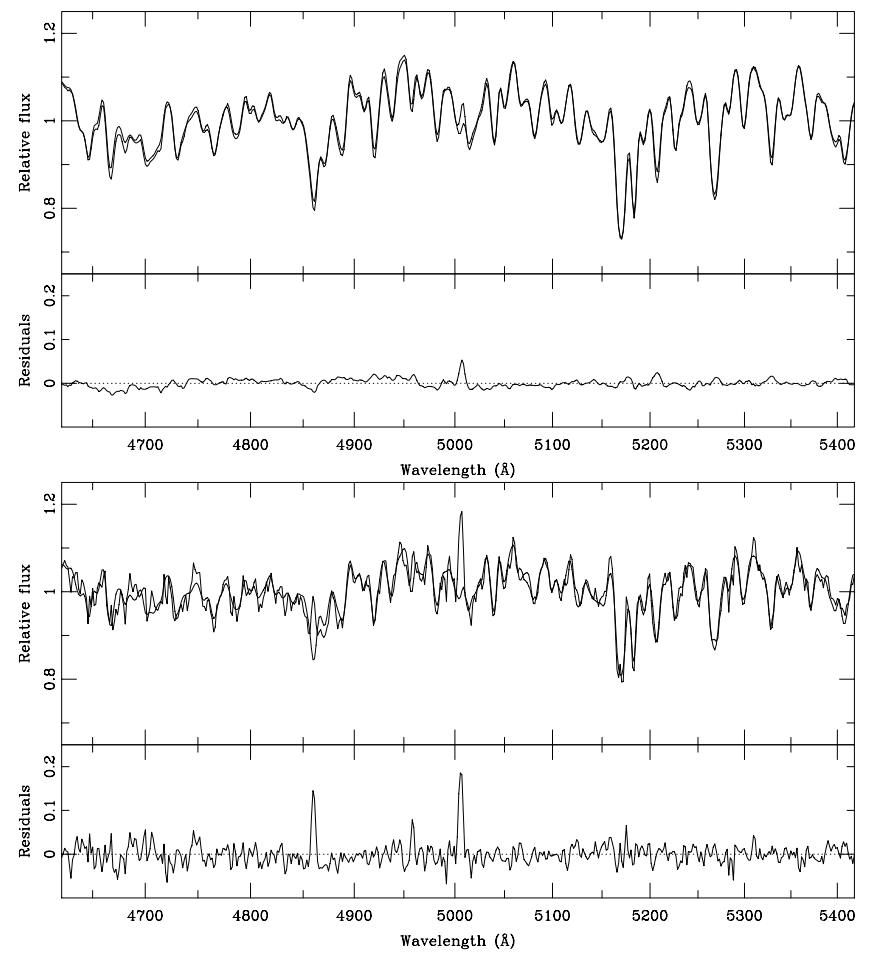

Fig. 2. Illustration of the velocity dispersion determinations using optimal templates for NGC 7332 (upper plot) and UGC 10043 (lower plot). For each plot, the upper panels show the observed galaxy spectrum (thick line) and the corrected optimal template spectrum (thin line). The residuals of the fits are shown in the lower panels.

different zenithal distances to check the atmospheric extinction correction. All the calibration curves of each run were averaged, and the flux calibration errors were estimated by the differences among the indices measured with different curves.

\section{(vi) Extraction of spectra}

Once the galaxy frames were fully reduced, we added all the frames of the same galaxies and extracted individual spectra along the slit. The availability of reliable error frames allowed us to compute the number of spatial bins to be coadded to guarantee a minimum $S / N$ in the final spectra. Using the prescriptions given in Cardiel et al. (1998), we chose a lower $S / N$ cutoff of 15 (per $\AA$; mean value along the full spectral range), which ensures a typical error in most of the atomic Lick indices below 20\%. For the outer spatial regions, we coadded some other spectra to get a minimum $S / N$ of 10 , which is enough to measure molecular indices (like $\mathrm{Mg}_{2}$ ) with a relative uncertainty below $10 \%$, even though it does lead to significant errors in the atomic indices. 
Table 3. Dynamical parameters of the bulge sample.

\begin{tabular}{|c|c|c|c|c|c|c|c|c|c|c|c|}
\hline Galaxy & $\overline{V_{\mathrm{r}}^{a}}$ & $\Delta \Delta V_{\mathrm{r}}$ & $V_{\mathrm{rot}}^{b}$ & $\Delta V_{\text {rot }}$ & $\overline{\overline{\sigma_{0}^{c}}}$ & $\Delta \sigma_{0}$ & $\langle\sigma\rangle^{d}$ & $\overline{\Delta \Delta\langle\sigma\rangle}$ & $\overline{\sigma_{\max }^{e}}$ & $\overline{\sigma_{\text {ref }}}$ & $\overline{\operatorname{Ref}^{f}}$ \\
\hline NGC 522 & 2719.0 & 12.5 & 12.1 & 4.9 & 82.1 & 9.3 & 88.2 & 8.8 & 99 & & \\
\hline NGC 585 & 5358.4 & 6.8 & 5.5 & 4.5 & & & 174.3 & 4.7 & 188 & & \\
\hline NGC 678 & 2814.9 & 15.0 & 13.3 & 6.3 & 163.4 & 2.1 & 170.7 & 4.1 & 183 & & \\
\hline NGC 891 & 548.2 & 12.3 & 1.9 & 2.2 & & & 95.4 & 5.1 & 95 & 72 & (2) \\
\hline NGC 973 & 4081.0 & 9.6 & 1.5 & 6.0 & & & 173.3 & 9.3 & 226 & & \\
\hline NGC 1032 & 2702.9 & 7.7 & 16.3 & 9.4 & & & 214.2 & 2.8 & 225 & & \\
\hline NGC 1184 & 2278.2 & 8.0 & 5.1 & 3.1 & 230.3 & 3.0 & 219.6 & 7.6 & 234 & 229 & (3) \\
\hline NGC 1351A & 1329.3 & 17.6 & 6.1 & 15.4 & & & 141.4 & 28.5 & 141 & & \\
\hline NGC 1886 & 1689.8 & 15.1 & 8.0 & 3.3 & & & 111.0 & 6.5 & 120 & & \\
\hline NGC 3957 & 1589.7 & 10.2 & 1.6 & 2.2 & & & 131.4 & 5.3 & 149 & & \\
\hline NGC 5084 & 1671.6 & 16.8 & 10.5 & 9.5 & 207.6 & 1.7 & 200.0 & 4.0 & 214 & 215 & (1) \\
\hline NGC 6010 & 2058.5 & 3.3 & 2.9 & 3.3 & 148.4 & 1.2 & 146.7 & 4.9 & 157 & 144 & (4) \\
\hline NGC 6829 & 3303.3 & 11.0 & 1.2 & 5.6 & & & 118.1 & 6.3 & 130 & & \\
\hline NGC 7183 & 2594.9 & 12.8 & 2.2 & 5.0 & & & 138.8 & 5.3 & 157 & & \\
\hline NGC 7264 & 4210.1 & 14.4 & 6.9 & 4.6 & & & 165.6 & 6.9 & 205 & & \\
\hline NGC 7332 & 1206.3 & 2.2 & 2.6 & 2.8 & 117.2 & 0.6 & 116.1 & 2.8 & 122 & 134 & (1) \\
\hline NGC 7396 & 4882.5 & 12.2 & 8.9 & 6.6 & & & 220.6 & 4.1 & 224 & & \\
\hline NGC 7703 & 3970.5 & 7.1 & 7.1 & 4.8 & 149.9 & 3.2 & 148.5 & 5.9 & 154 & 166 & (3) \\
\hline NGC 7814 & 1051.4 & 6.9 & 4.8 & 4.5 & & & 170.3 & 2.4 & 187 & 172 & (1) \\
\hline IC 1711 & 2785.2 & 11.8 & 9.0 & 5.2 & & & 122.3 & 12.2 & 161 & & \\
\hline IC 1970 & 1300.8 & 7.2 & 11.6 & 10.6 & & & 82.8 & 12.1 & 95 & & \\
\hline IC 2531 & 2386.8 & 11.7 & 3.8 & 4.1 & & & 126.6 & 8.7 & 127 & & \\
\hline IC 5176 & 1739.3 & 10.5 & 4.1 & 4.3 & & & 152.7 & 6.4 & 160 & & \\
\hline IC 5264 & 2025.1 & 6.3 & 2.6 & 3.6 & & & 101.1 & 3.4 & 106 & & \\
\hline UGC 10043 & 2180.3 & 16.0 & 8.2 & 6.4 & & & 88.5 & 15.7 & 89 & & \\
\hline UGC 11552 & 4479.5 & 11.1 & 2.7 & 11.3 & & & 151.3 & 10.7 & 167 & & \\
\hline UGC 11587 & 4481.5 & 7.8 & 2.3 & 4.9 & 213.0 & 2.9 & 203.4 & 8.5 & 224 & & \\
\hline ESO 079-003 & 2613.5 & 8.1 & 1.5 & 6.8 & & & 120.7 & 8.5 & 160 & & \\
\hline ESO 234-053 & 6232.9 & 16.0 & 0.3 & 4.9 & 204.9 & 3.1 & 200.4 & 7.6 & 226 & & \\
\hline ESO 311-012 & 1106.8 & 10.7 & 3.2 & 5.3 & 124.2 & 3.5 & 132.1 & 4.0 & 147 & & \\
\hline ESO 443-042 & 2815.8 & 24.8 & 8.7 & 9.6 & & & 118.6 & 14.9 & 119 & & \\
\hline ESO 512-012 & 3313.4 & 19.0 & 13.0 & 6.5 & & & 122.8 & 12.4 & 123 & & \\
\hline
\end{tabular}

${ }^{a}$ Mean radial velocity (in $\mathrm{km} \mathrm{s}^{-1}$ ). ${ }^{b}$ Maximum rotational velocity (see the text). ${ }^{c}$ Central velocity dispersion. ${ }^{d}$ Mean velocity dispersion. ${ }^{e}$ Maximum velocity dispersion (see the text). ${ }^{f}$ References are: (1) Average value quoted in HYPERLEDA; (2) Bottema et al. (1991); (3) Di Nella et al. (1995), and (4) Falcón-Barroso et al. (2002).

\section{Dynamical parameters}

Radial velocities and velocity dispersions for each spectrum along the bulge radii were measured using a dedicated program within $\mathrm{R}_{\mathrm{E}} \mathrm{D}_{\mathrm{m}}^{\mathrm{uc}} \mathrm{E}$, which incorporates the MOVEL and OPTEMA algorithms devised by González (1993). The MOVEL procedure is a refined version of the classic Fourier quotient method (Sargent et al. 1977). The basic improvement over the standard procedure is that, starting from a first guess of $\gamma$ (mean line-strength), $V$ (radial velocity), and $\sigma$ (velocity dispersion), it creates a model galaxy that is processed in parallel to the galaxy spectrum. The differences between the input and output parameters of the model spectrum are then used to correct the galaxy power spectrum from systematic effects in the handling of data in the Fourier space. The OPTEMA algorithm allowed us to overcome the typical template mismatch problem. In order to do this, a number of stellar spectra of different spectral types and luminosity classes is fed into the program. In our case, we combined our template stars to construct representative input spectra of the following types: A3-5V, G0-2V, G5V, K0$1 \mathrm{~V}$, G8-K0III, K3III, K5-7III, and M3III. The algorithm then constructs an optimal template as a linear combination of the above template spectra, which minimizes the residuals between the galaxy spectrum and the broadened template. Meanwhile, the dynamical parameters are derived as part of the same minimization process. Our tests showed that if a single template star is used to measure the velocity dispersion (instead of the above combination), systematic errors as large as $10 \%$ can be introduced.

Figure 2 illustrates the result of this procedure for two galaxies (NGC 7332 and UGC 10043), showing, in the upper panels, the fits between the observed central spectra of the galaxies and the corresponding optimal templates corrected with the derived dynamical parameters. The lower panels show the residual of the fits. Note that emission lines, although almost hidden in the original spectrum (like the [O III] $\lambda 5007$ line for NGC 7332) are clearly detected after subtracting the template. (Once an emission line is detected, the corresponding wavelength region is not used for minimizing the residuals.) In fact, the optimal templates derived for each galaxy were later used to mask the regions possibly containing emission lines.

Realistic errors in the derived parameters (radial velocity and velocity dispersion) were computed by performing Monte-Carlo simulations, repeating the whole process (including the derivation of the optimal template) for a large number of simulated galaxy spectra created using the error spectrum obtained during the reduction process. We checked that, in order to measure velocity dispersion of the order of the spectral resolution $\left(\sim 100 \mathrm{~km} \mathrm{~s}^{-1}\right.$ for runs $1,2,4$, and 5) with a relative error below $20 \%$, we needed spectra with $S / N$ (per $\AA$ ) greater than 15 . This coincides with the cutoff value for the spectral extraction to measure the atomic indices.

Once an optimal template is derived for the central or maximum $S / N$ (if the center is affected by dust lanes) spectrum, the 


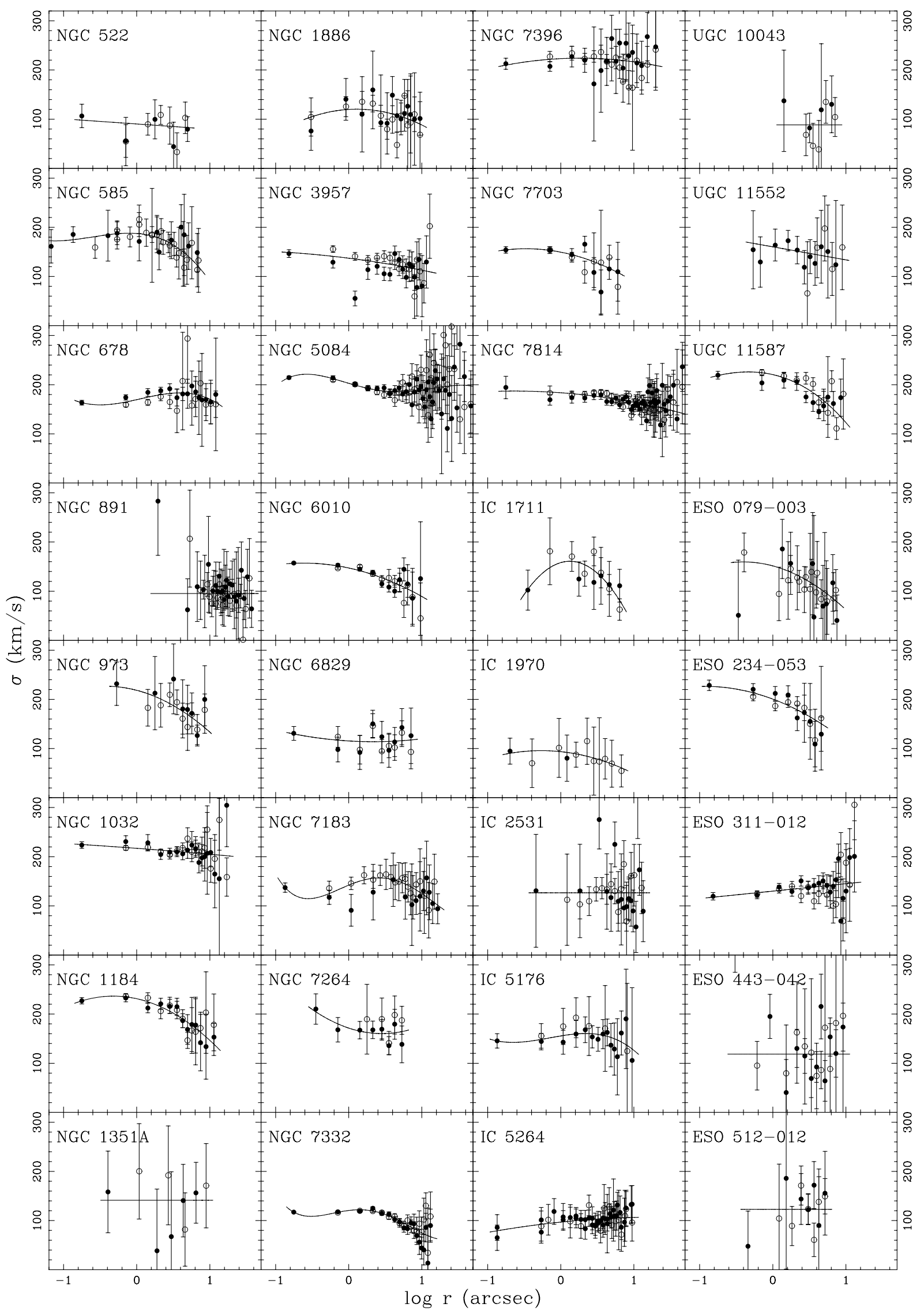

Fig. 3. Velocity dispersion profiles for the galaxy sample. Filled and open symbols refer to spectra of the bulges at either side of the galaxy centers. The plotted lines represent polynomial fits to the profiles. See text for details. 
procedure is repeated for all the spectra along the bulge radii, deriving radial profiles for the radial velocity and velocity dispersion. Note that the template derived for the central spectrum is used for the analysis of the rest of the spectra and, therefore, that we are not taking the possible effect of population gradients in the derived sigma profiles into account. However, we preferred to introduce this small inaccuracy over artificially increasing the scatter of the parameters profiles due to unreal changes in the derived linear combinations of templates at each radius. Note also that any radial change in mean line-strength (i.e. $\gamma$ ) or continuum shape is properly corrected from with the MOVEL algorithm.

The dynamical parameters and their corresponding errors at each galactocentric radius are listed in Table 3. Since the slit was oriented along the minor axes of the bulges, no rotation along the slit was expected. To check this, we include the maximum rotational velocity in Table 3 (computed as an error-weighted mean of the, generally two, spectra on each side of the galaxy center with the highest rotational velocity) and its error. It is apparent from the table that most bulges do not show any significant rotation along the minor axes. For some galaxies, however, we observed some hints of rotation or peculiar $V_{\mathrm{r}}$ profiles in the central regions, probably due to slight misalignments of the slit with respect to the minor axis direction or the bulge center.

For galaxies with no obvious dust lanes in the central regions, the table also includes the central velocity dispersion $\sigma_{0}$, measured in an aperture of 4 arcsec times the slit width centered on the galaxy center. We also list mean velocity dispersions $(\langle\sigma\rangle)$ defined as the error-weighted mean velocity dispersion of all the extracted spectra along the radius (which is very close to the velocity dispersion that is derived when adding all the spectra along the slit).

The derived velocity dispersion profiles for the galaxy sample are plotted in Fig. 3. Typically, the velocity dispersion decreases with galactocentric radius, although there are different behaviors and some galaxies exhibit a large scatter. It should be noted that the main motivation for measuring velocity dispersion profiles in this paper is to have the necessary information to carry out accurate corrections to the Lick/IDS indices (detailed below). Since line-strength indices depend on the spectral resolution, all spectra along the slit must be corrected to the same velocity dispersion before measuring the indices in order to avoid the introduction of spurious line-strength gradients. However, if we were to use the nominal velocity dispersion values obtained for each individual spectrum to perform this correction, the errors associated with the $\sigma$ measurement would be introduced into the measured indices. To avoid this, we smoothed the velocity dispersion profiles by fitting low-order polynomials to the data, and we used the predictions of these polynomials for correcting the indices to the desired velocity dispersions. Note that we are not pretending to assign any physical meaning to these polynomial radial profiles; they only serve a calibration purpose. The column $\sigma_{\max }$ in Table 3 gives the maximum velocity dispersion indicated by the polynomials in the observed radial range.

Finally, we compared the derived values with the available data from the literature (last columns of Table 3) to check for any systematic offset in the derived velocity dispersion. To minimize aperture differences, these reference data should be compared with our central $\left(\sigma_{0}\right)$ values when available, or else with $\langle\sigma\rangle$. It can be seen that we do not have any systematic offset relative to the scarce previous data.
Table 4. Coefficients of the polynomials to correct Lick indices for broadening effects.

\begin{tabular}{lcrr}
\hline \hline Index & \multicolumn{1}{c}{$c_{1}$} & \multicolumn{1}{c}{$c_{2}$} & \multicolumn{1}{c}{$c_{3}$} \\
\hline $\mathrm{H} \delta_{\mathrm{A}}$ & 0.76129 & 0.10120 & 0.13751 \\
$\mathrm{H} \delta_{\mathrm{F}}$ & 1.25993 & -0.51501 & 0.25508 \\
$\mathrm{CN}_{1}$ & 0.70442 & 0.31941 & -0.02382 \\
$\mathrm{CN}_{2}$ & 0.76492 & -0.00441 & 0.23949 \\
$\mathrm{Ca}_{2} 27$ & 0.78022 & -0.28090 & 0.50067 \\
$\mathrm{G} 4300$ & 0.89152 & 0.10002 & 0.00847 \\
$\mathrm{H} \gamma_{\mathrm{A}}$ & 1.11845 & -0.21248 & 0.09403 \\
$\mathrm{H} \gamma_{\mathrm{F}}$ & 0.80429 & 0.25651 & -0.06080 \\
$\mathrm{Fe} 4383$ & 0.87985 & -0.00952 & 0.12967 \\
$\mathrm{Ca} 4455$ & 0.63849 & 0.09619 & 0.26532 \\
$\mathrm{Fe} 4531$ & 0.91332 & -0.01235 & 0.09903 \\
$\mathrm{Fe} 4668$ & 1.00545 & -0.12685 & 0.12140 \\
$\mathrm{H} \beta$ & 0.99068 & 0.00563 & 0.00369 \\
$\mathrm{Fe} 5015$ & 0.78157 & 0.17915 & 0.03928 \\
$\mathrm{Mg}_{1}$ & 0.97728 & 0.01066 & 0.01206 \\
$\mathrm{Mg}_{2}$ & 0.98082 & 0.02199 & -0.00282 \\
$\mathrm{Mgb}_{\mathrm{Fe} 5270}$ & 0.96450 & -0.07495 & 0.11045 \\
$\mathrm{Fe} 5335$ & 0.84333 & 0.12283 & 0.05184 \\
$\mathrm{Fe} 5406$ & 0.89017 & -0.08141 & 0.23817 \\
$\mathrm{Fe} 5709$ & 0.89437 & 0.01365 & 0.25104 \\
$\mathrm{Fe} 5782$ & 0.86475 & -0.14458 & 0.09198 \\
$\mathrm{Na} 58950.94445$ & 0.01617 & 0.03938 \\
$\mathrm{TiO}_{1}$ & 1.00000 & 0.00000 & 0.00000 \\
$\mathrm{TiO}_{2}$ & 1.00000 & 0.00000 & 0.00000 \\
\hline
\end{tabular}

\section{Line-strength indices}

Line-strength indices in the Lick/IDS system (e.g. Worthey et al. 1994; Worthey \& Ottaviani 1997) were measured for all the extracted spectra along the measured extent of the bulge. We measured all the indices from $\mathrm{H} \delta$ to Fe5406. In the case of runs 4 and 5 (cf. Table 2), the extended spectral range allowed us to also include the redder Lick indices (up to the TiO indices). The next sections explain all the procedures to derive reliable errors in the line-strengths and to transform them to the spectrophotometric Lick/IDS system. All these steps were possible thanks to including a number of stars from the Lick/IDS library in the different observing runs. In particular, we observed 40, 40, 19, 4, and 6 Lick standard stars (covering a range of spectral types and luminosity classes) in runs from 1 to 5 , respectively.

\subsection{Error analysis}

A first estimation of errors in the line strengths was accomplished by taking three different error sources into account: (i) photon noise (the errors were calculated using the variance spectra computed as part of the reduction process); (ii) flux calibration (the estimation was based on the comparison of the indices measured using the different flux calibration curves obtained in each observing run); and (iii) uncertainties in the wavelength scale (due to errors in the wavelength calibration and in the measurement of the radial velocity). Except for the spectra with the highest $S / N$, the dominant error source was the first one.

To check and refine the error analysis, we analyzed the line strengths obtained for $(i)$ repeated observations of the same stars within each observing run, (ii) stars in common among different observing runs (e.g. all the 40 Lick stars observed in run 1 were repeated in run 2), and (iii) separate observations of the same galaxies within each run. (Typically the observation of a galaxy was split into 4-12 different exposures.) Using this 
Table 5. Spectral resolutions, residual errors, and offsets needed to transform line-strength indices to the Lick/IDS system.

\begin{tabular}{|c|c|c|c|c|c|c|c|c|c|c|c|}
\hline \multirow[t]{2}{*}{ Index } & $\overline{\overline{\sigma_{\mathrm{L}}^{a}}}$ & \multicolumn{4}{|c|}{ Residual errors } & \multicolumn{4}{|c|}{ Offsets } & \multicolumn{2}{|c|}{$\sigma^{b}$} \\
\hline & $\mathrm{km} \mathrm{s}^{-1}$ & run 1 & run 2 & run 3 & runs 4,5 & run 1 & run 2 & run 3 & runs 4,5 & $\mathrm{rms}$ & $\exp$ \\
\hline$\overline{\mathrm{H} \delta_{\mathrm{A}}}$ & 325 & 0.00 & 0.00 & 0.00 & 0.20 & -0.34 & -0.34 & 0.00 & -1.09 & 0.66 & 0.37 \\
\hline $\mathrm{H} \delta_{\mathrm{F}}$ & 325 & 0.00 & 0.00 & 0.00 & 0.00 & -0.12 & -0.09 & 0.00 & -0.81 & 0.35 & 0.24 \\
\hline $\mathrm{CN}_{1}$ & 325 & 0.005 & 0.005 & 0.005 & 0.008 & +0.003 & +0.003 & -0.009 & 0.000 & 0.020 & 0.015 \\
\hline $\mathrm{CN}_{2}$ & 325 & 0.004 & 0.004 & 0.004 & 0.011 & +0.008 & +0.008 & -0.015 & -0.011 & 0.021 & 0.016 \\
\hline $\mathrm{Ca} 4227$ & 300 & 0.04 & 0.04 & 0.04 & 0.00 & 0.00 & 0.00 & -0.08 & 0.00 & 0.23 & 0.17 \\
\hline G4300 & 300 & 0.13 & 0.13 & 0.13 & 0.00 & -0.08 & -0.08 & 0.00 & 0.00 & 0.38 & 0.32 \\
\hline $\mathrm{H} \gamma_{\mathrm{A}}$ & 275 & 0.19 & 0.19 & 0.21 & 0.00 & +0.41 & +0.41 & +0.41 & +0.34 & 0.48 & 0.40 \\
\hline $\mathrm{H} \gamma_{\mathrm{F}}$ & 275 & 0.04 & 0.00 & 0.00 & 0.00 & +0.17 & +0.17 & 0.00 & +0.10 & 0.22 & 0.19 \\
\hline $\mathrm{Fe} 4383$ & 250 & 0.22 & 0.21 & 0.21 & 0.09 & +0.08 & +0.08 & 0.00 & -0.36 & 0.53 & 0.45 \\
\hline Ca4455 & 250 & 0.04 & 0.04 & 0.04 & 0.00 & +0.32 & +0.32 & 0.00 & +0.28 & 0.20 & 0.20 \\
\hline $\mathrm{Fe} 4531$ & 250 & 0.10 & 0.10 & 0.10 & 0.18 & +0.15 & +0.15 & -0.32 & 0.00 & 0.35 & 0.31 \\
\hline Fe4668 & 250 & 0.15 & 0.19 & 0.15 & 0.57 & 0.00 & 0.00 & -1.03 & 0.00 & 0.57 & 0.59 \\
\hline $\mathrm{H} \beta$ & 225 & 0.10 & 0.10 & 0.10 & 0.04 & -0.07 & -0.07 & 0.00 & -0.14 & 0.22 & 0.20 \\
\hline Fe5015 & 200 & 0.15 & 0.19 & 0.15 & 0.21 & +0.26 & +0.26 & -0.11 & 0.00 & 0.64 & 0.40 \\
\hline $\mathrm{Mg}_{1}$ & 200 & 0.004 & 0.004 & 0.004 & 0.007 & +0.011 & +0.003 & +0.016 & +0.009 & 0.011 & 0.010 \\
\hline $\mathrm{Mg}_{2}$ & 200 & 0.006 & 0.005 & 0.003 & 0.005 & +0.025 & +0.020 & +0.021 & +0.016 & 0.012 & 0.010 \\
\hline Mgb & 200 & 0.04 & 0.03 & 0.02 & 0.07 & 0.00 & 0.00 & -0.08 & 0.00 & 0.31 & 0.17 \\
\hline $\mathrm{Fe} 5270$ & 200 & 0.00 & 0.00 & 0.00 & 0.11 & 0.00 & +0.03 & 0.00 & 0.00 & 0.23 & 0.16 \\
\hline Fe5335 & 200 & 0.02 & 0.02 & 0.02 & 0.16 & -0.06 & -0.06 & 0.00 & +0.18 & 0.26 & 0.16 \\
\hline Fe5406 & 200 & 0.04 & 0.04 & 0.04 & 0.03 & -0.04 & -0.04 & -0.19 & 0.00 & 0.23 & 0.14 \\
\hline Fe5709 & 200 & & & & 0.00 & & & & -0.08 & 0.14 & 0.14 \\
\hline $\mathrm{Fe} 5782$ & 200 & & & & 0.00 & & & & +0.14 & 0.10 & 0.13 \\
\hline Na5895 & 200 & & & & 0.24 & & & & 0.00 & 0.26 & 0.40 \\
\hline $\mathrm{TiO}_{1}$ & 200 & & & & 0.003 & & & & +0.013 & 0.008 & 0.007 \\
\hline $\mathrm{TiO}_{2}$ & 200 & & & & 0.000 & & & & -0.015 & 0.005 & 0.004 \\
\hline
\end{tabular}

${ }^{a}$ Spectral resolutions at which line-strength indices should be measured to roughly match the original Lick/IDS system. ${ }^{b}$ Measured (rms) and expected (exp) standard deviations for the comparison of indices with the original values of the Lick/IDS system.

data, we computed residual errors as the quadratic differences between the measured rms dispersions and the expected total error from the three sources of uncertainty quoted in the previous paragraph. The resulting residual errors are listed in Table 5 for each index and observing run. They are relatively modest but were included anyway in the final uncertainties.

\subsection{Emission-line corrections}

Some line-strength indices, in particular $\mathrm{H} \beta, \mathrm{Fe} 5015$, and $\mathrm{Mgb}$, are potentially affected by emission lines (e.g., Goudfrooij \& Emsellem 1996). Most of our galaxies exhibit non-negligible nebular emission, as judged from their [O III $] \lambda 5007$ line. Usually, the emission is concentrated towards the bulge centers, where the spectra are heavily contaminated by the disk light (H II regions can project onto the slit). However, in some special cases (e.g., NGC 1886 and NGC 7332), line emission can even be detected in the outer part of the bulge, where any contamination by disk light is negligible. For instance, in NGC 7332, the line emission increases to $E W([\mathrm{O}$ III $] \lambda 5007) \simeq 4 \AA$ at a distance of $\sim 16$ arcsec from the center.

The correction of $\mathrm{H} \beta$ from the emission contribution is a troublesome task. The usual procedure (see eg. González 1993; Trager et al. 2000) relies on using the equivalent width of [O III] to apply an empirical correction. In this paper, given the uncertainties of that correction and the fact that the emission is relatively strong for many of the galaxies, we have decided not to correct $\mathrm{H} \beta$ and, therefore, not to use it in our analysis in the cases where any [O III] $\lambda 5007$ emission was detected (in particular when its measured equivalent width was larger than its error).

We determined equivalent widths of [O III] $\lambda 5007$ after subtracting from each galaxy spectrum an emission-free template spectrum constructed from the broadened optimal template derived for that galaxy during the velocity dispersion measurement (see Fig. 2). In order to measure the Fe5015 and Mgb indices in spectra with emission lines, we masked the spectral regions affected by the [O III] $\lambda 4959,[\mathrm{O}$ III] $\lambda 5007$, and [N I] $\lambda 5199$ lines and replaced them with the corresponding regions in the broadened optimal template spectrum.

\subsection{Broadening corrections}

As mentioned before in Sect. 5, the Lick/IDS indices have to be corrected for spectral broadening. In order to avoid the introduction of artificial line-strength gradients due to changes in velocity dispersion along the slit, we broadened all the spectra of a given galaxy to match the maximum velocity dispersion $\sigma_{\max }$ derived in Sect. 5 by convolving it with a Gaussian of width $\left(\sigma_{\max }^{2}-\sigma_{r}^{2}\right)^{1 / 2}$, where $\sigma_{r}$ is the velocity dispersion at each radius predicted by the corresponding polynomial fit.

The conversion of the line indices to the resolution of the Lick system (see Sect. 6.4) was done as follows. In the cases where the total resolution of the spectra (the quadratic sum of instrumental resolution and the maximum velocity dispersion for the galaxy) was below the Lick resolution, we simply broadened the spectra by the quadratic difference. In other cases, we applied an empirical correction to the measured indices. This correction was derived by broadening stellar spectra by convolution with Gaussians having a range of $\sigma$ values. Since this correction depends on the individual spectral type of the star, we instead used the optimal templates obtained in the velocity dispersion measurement procedure to derive the following expression:

$I\left(\sigma_{0}\right)=I(\sigma)\left(c_{1}+c_{2} \sigma / \sigma_{0}+c_{3}\left(\sigma / \sigma_{0}\right)^{2}\right)$ 

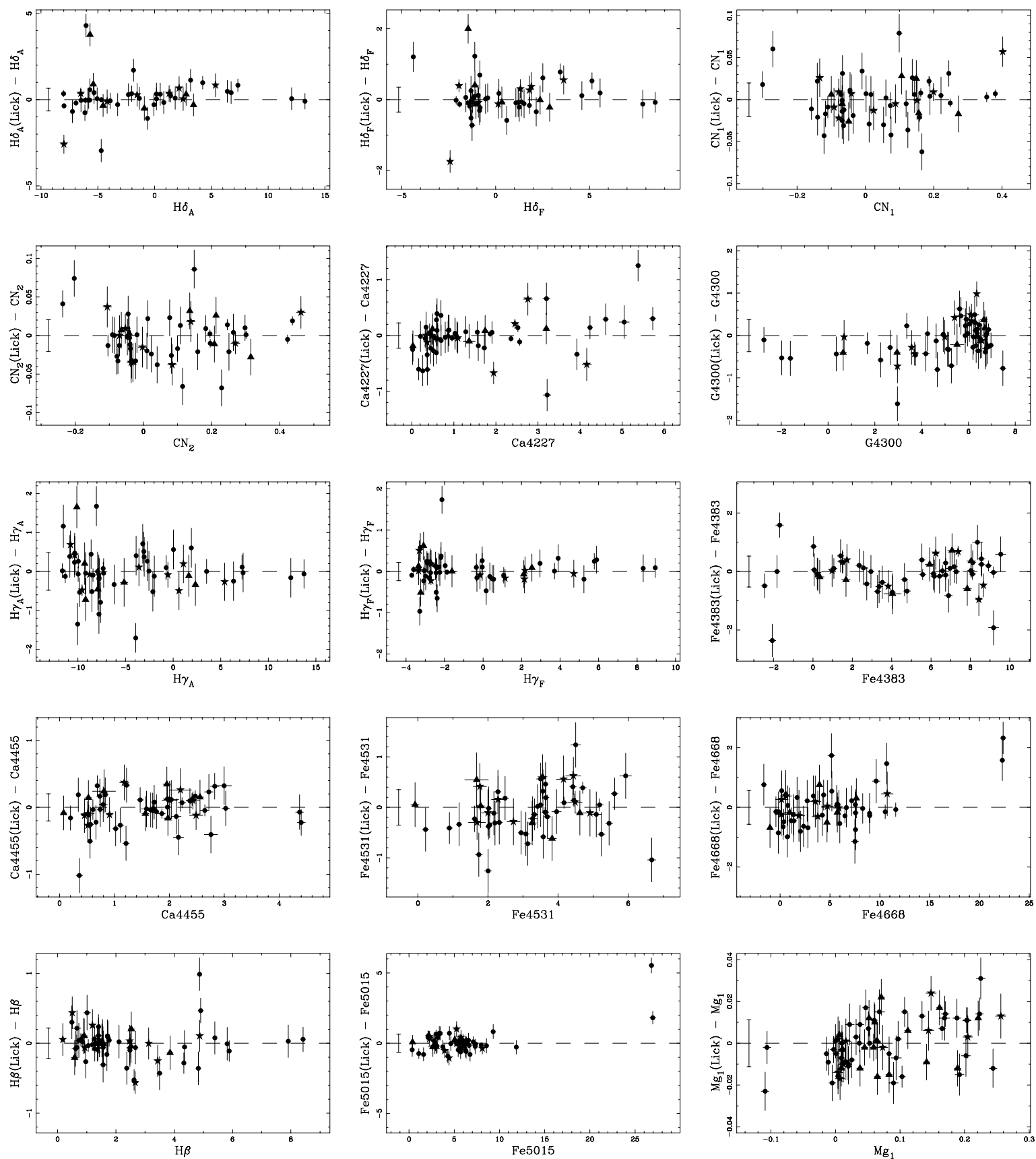

Fig. 4. Comparison of the line-strength indices in the Lick/IDS spectra and the measurements in our sample of stars after correcting to the Lick/IDS system. Different symbols refer to the different observing runs, using the following code: circles for runs 1 and 2, triangles for run 3, and stars for runs 4 and 5. In the case of the $\mathrm{TiO}$ indices, both $\mathrm{TiO}_{1}$ are $\mathrm{TiO}_{2}$ are plotted in the same panel, using closed and open symbols for both indices, respectively. The length of the error bar near the left end of each panel is twice the rms standard deviation of each dataset with respect to the zero offset horizontal line.

which can be used to convert an index $I$ measured at a resolution $\sigma$ to any other resolution $\sigma_{0}$. The values of the $c_{i}$ coefficients are listed in Table 4 for all the Lick indices. In principle, the exact values of the coefficients depend on the particular optimal template used. However, the spectral differences among them have a weak effect on the derived polynomials and the uncertainties introduced by using the listed mean coefficients are well below the other sources of errors.

\subsection{Conversion to the Lick/IDS system}

Transformation of line strengths to the Lick/IDS system is not straightforward. Basically, there are two important effects that have to be taken into account. First, the Lick/IDS spectra are not flux-calibrated using a spectrophotometric standard star, but by using a normalized tungsten lamp spectrum. Thus, there are significant differences in the spectral shapes when compared with flux-calibrated spectra. This effect causes systematic offsets, especially for indices with broad bands like (e.g.) $\mathrm{Mg}_{2}$. Second, the spectral resolution of the Lick spectra is not constant with wavelength, in particular it degrades considerably towards the blue end. This has an important effect on narrow-line indices, like many of the atomic ones. For a comprehensive discussion of these effects, we refer the reader to Worthey \& Ottaviani (1997).

To transform our data to the Lick/IDS system, we performed the following steps. First, we estimated the resolution at which 

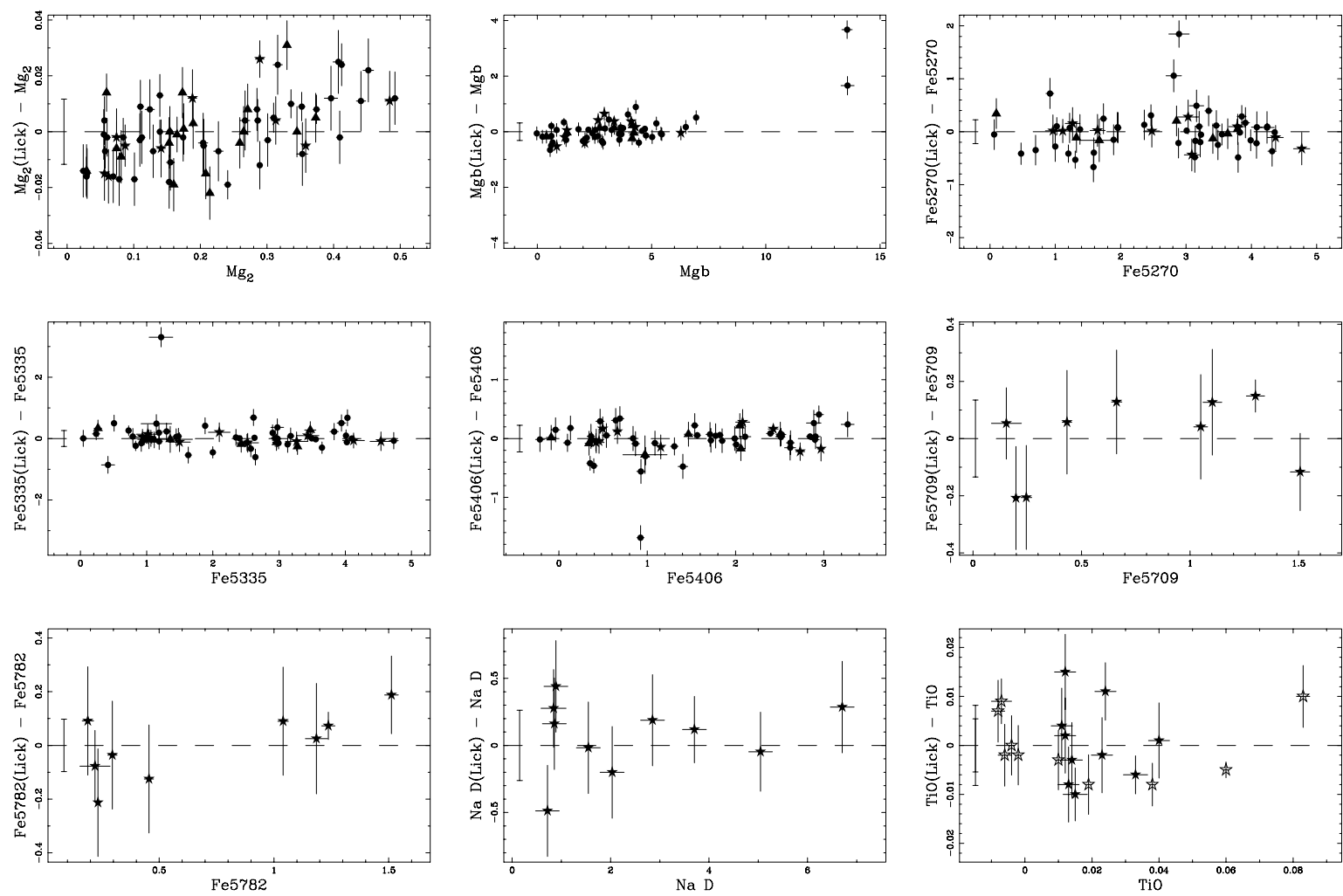

Fig. 4. continued.

each particular index should be measured by broadening our large set of stars in common with the Lick library to several line widths, in steps of $25 \mathrm{~km} \mathrm{~s}^{-1}$. We did not attempt to find precise resolutions for each index since these depend on the particular set of stars being used. Instead, we estimated the approximate line widths $\sigma_{\mathrm{L}}$ which, changing softly with wavelength, minimized the residuals. These are given, for each index, in the second column of Table 5. These resolutions agree with the rough estimates given in Worthey \& Ottaviani (1997).

We then broadened all our stellar spectra to the above $\sigma_{\mathrm{L}}$ resolutions and measured the line-strength indices. The comparison of these measurements with those in the original Lick/IDS spectra allowed us to derive mean offsets for all the indices in each observing run. These additive offsets, listed in Table 5, are mainly due to differences in flux calibration between both systems, but also include a fine tuning of the broadening corrections and systematic effects in both our data (like uncertainties in the spectral resolution or flux calibration) and the Lick/IDS data (like spectral peculiarities that depend on the particular Lick/IDS run in which each star was observed). Note that we obtain different offsets for each observing run (although in most cases they are similar). In fact, the comparison with Lick data provides us with an anchor system to correct for small offsets among the different runs. It must be noted that the offsets for run 3 , with a significantly lower spectral resolution, are of the same order as the offsets for the other runs, which confirms that the broadening corrections have been properly done.

Figure 4 shows a comparison of the line-strength indices in the Lick/IDS spectra and the measurements in our sample of stars after correcting to the Lick/IDS system. Error bars were derived by combining the computed errors for our measurements and the errors of the original Lick/IDS indices as taken from Worthey et al. (1994). The last two columns of Table 5 give the rms standard deviation with respect to the zero offset and the standard deviation that should be expected from the computed errors in the offsets. As can be seen, the former are usually larger than the latter, indicating that the errors in the $I$ (Lick) - $I$ (here) differences are somewhat underestimated. Given all the tests that we have carried out with our data, we think that this disagreement is most likely due to an underestimate of the errors in the original Lick measurements.

\subsection{Final data}

Once all the previous corrections had been derived, we applied them to the extracted spectra along the bulge radii and measured the line strengths. We derived two sets of final data:

1. In the first one, we introduced the residual errors, masked the emission lines, converted all the indices to a resolution corresponding to the maximum velocity dispersion along the radius (as listed in Table 3), and applied relative offsets among runs to put them in the same spectrophotometric system (which should be very close to a true flux-calibrated system). To do this, we applied the offsets listed in Table 5 with respect to run 2. (Offsets for run 2 were therefore zero.) However, we did not transform the indices to the Lick/IDS system. This data can be very useful for future studies working at any spectral resolution and in a flux-calibrated system. Note that, in this dataset, indices from different galaxies cannot be compared directly, since indices are measured at different resolutions.

2. For the second dataset, we carried out a full transformation to the Lick/IDS system, applying (in addition to the corrections from the last paragraph) the necessary offsets and the index-dependent velocity dispersion corrections. For this last step, we broadened the spectra when possible or applied the polynomials of Table 4 when the original resolutions (the quadratic sum of $\sigma_{\max }$ and the instrumental resolution) were higher than $\sigma_{\mathrm{L}}$ (Table 5). 

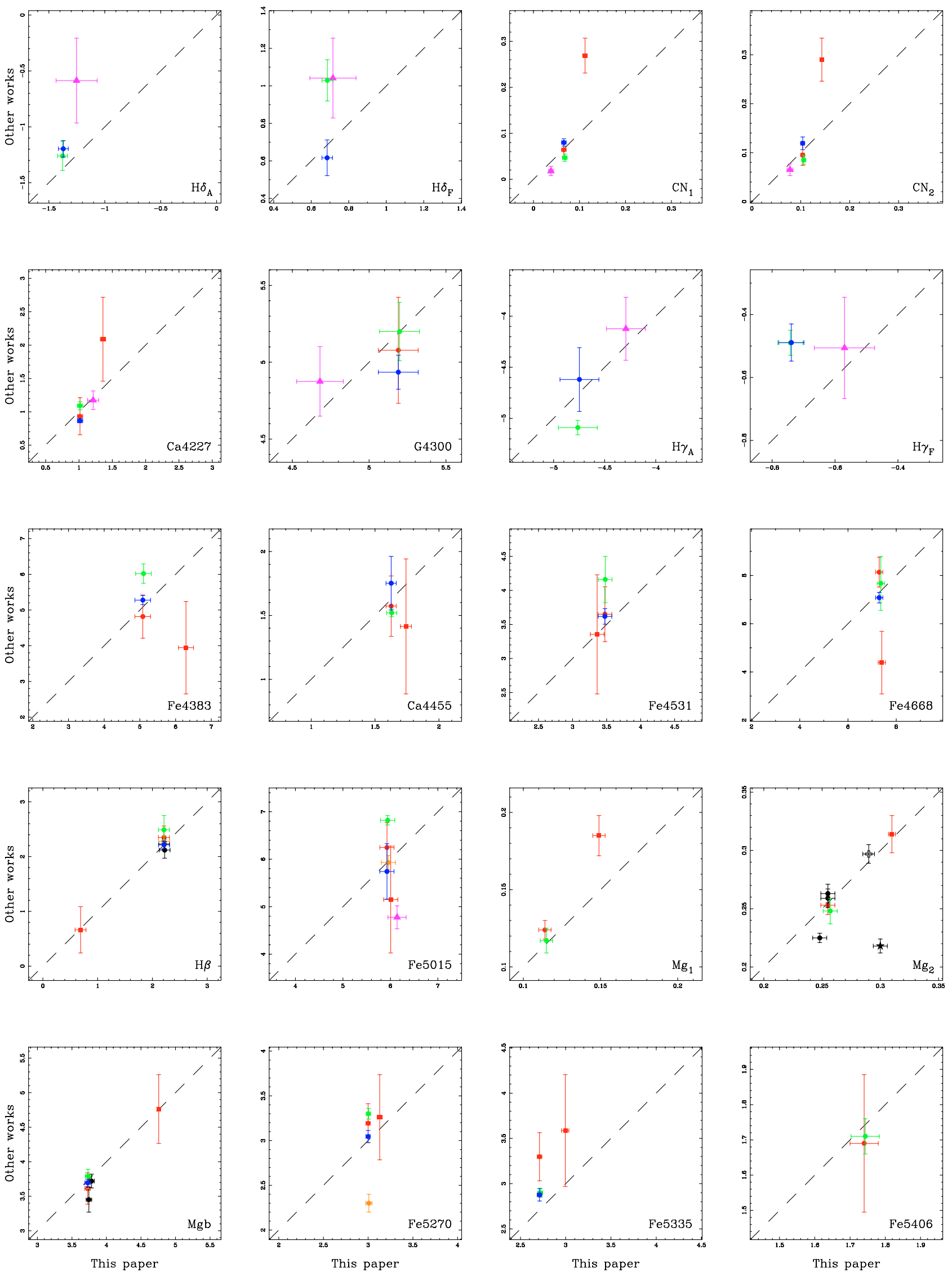

Fig. 5. Comparison of the line-strength indices measured in the central regions with data from other workers. Different symbols are used for different galaxies: squares for NGC 5084, crosses for NGC 6010, triangles for NGC 7703, circles for NGC 7332, and stars for NGC 7814. Color codes refer to data from Trager et al. (1998) (red), Sánchez-Blázquez et al. (2006) (blue), Caldwell et al. (2003) (magenta), Kuntschner et al. (2007) (orange), Denicoló et al. (2005) (green), and other sources (black). 


\subsection{Comparison with other works}

Finally, in Fig. 5 we present a comparison of the line-strengths measured in the central regions of our bulges with the available results from the literature. The galaxies with existing published data and the corresponding references are the following: NGC 5084 (Trager et al. 1998), NGC 6010 (Falcón-Barroso et al. 2002), NGC 7703 (Caldwell et al. 2003), NGC 7332 (Bender et al. 1993; Trager et al. 1998; Golev et al. 1999; FalcónBarroso et al. 2002, 2004; Denicoló et al. 2005; Sil' chenko 2006; Kuntschner et al. 2006; Sánchez-Blázquez et al. 2006b), and NGC 7814 (Prugniel et al. 2001). To perform the comparison for each galaxy and reference, we binned our spectra in the central regions of the bulges to reproduce the aperture used by the corresponding works as closely as possible. It is apparent from the figure that, although there is general agreement with the data from other workers, there are also some important discrepancies for some particular line strengths and authors (like some indices for NGC 5084 from Trager et al. 1998, or the $\mathrm{Mg}_{2}$ measurement for NGC 7814). These differences could be due to: low $S / N$ ratios in the spectra of other authors, variations in the centering of the slit or in the observed aperture, or differences in the calibration and data reduction processes. However, the fact that, when there are several published values for a galaxy (NGC 7332), our measurements agree with the majority of them gives us confidence in our measured values. For instance, for the 5 previous measurements for the central $\mathrm{Mg}_{2}$ index in NGC 7332, our value agrees with 4 out of them within the errors.

The comparison with the central line-strengths from Sánchez-Blázquez et al. (2006b) is of particular relevance, since in Paper II we will make use of this dataset as a comparison sample of elliptical galaxies. In this case, we plot the indices measured in an aperture of $2 \times 4$ arcsec (as listed in SánchezBlázquez 2004), finding good agreement with our data (with the exception of the $\mathrm{H} \gamma_{\mathrm{F}}$ index, although note that the results agree for $\left.\mathrm{H} \gamma_{\mathrm{A}}\right)$.

\section{Summary}

We have introduced our long-term project devoted to understanding the evolutionary status of galaxy bulges by presenting the sample and the most relevant details of our survey of long-slit spectra along the minor axis of bulges in edge-on spiral galaxies. After summarizing a data reduction process characterized by a very detailed error analysis, we have presented our procedures to measure dynamical parameters and all the Lick/IDS line-strength indices available in the spectra. A special emphasis was made in the broadening corrections of the indices and their conversion to the Lick/IDS system. As a final product, we derived tables giving all the parameters (radial velocities, velocity dispersions, and indices) at each galactocentric radius for all the bulges in the sample. In the case of the indices, two datasets have been obtained: one with the indices in flux-calibrated spectra and at the natural resolution of the bulges (i.e. internal plus instrumental), and another in which all the indices have been converted to the Lick/IDS system. These two datasets will be available in electronic form at CDS.

In the next paper of this series we will measure line-strength gradients and analyze them in the light of different galaxy formation scenarios.

Acknowledgements. We are grateful to Nicolas Cardiel for his help in technical issues in the reduction process. The INT is operated on the island of La Palma by the Royal Greenwich Observatory at the Observatorio del Roque de los Muchachos of the Instituto de Astrofisica de Canarias. This work was supported by the Spanish research project AYA 2003-01840.

\section{References}

Andredakis, Y. C., Peletier, R. F., \& Balcells, M. 1995, MNRAS, 275, 874 Arimoto, N., \& Jablonka, P. 1991, A\&A, 249, 374

Balcells, M., \& Peletier, R. F. 1994, AJ, 107, 135

Balcells, M., Graham, A. W., Domínguez-Palmero, L., \& Peletier, R. F. 2003, ApJ, 582, L79

Bekki, K., \& Shioya, Y. 1999, ApJ, 513, 108

Bender, R., Burstein, D., \& Faber, S. M. 1992, ApJ, 399, 462

Bender, R., Burstein, D., \& Faber, S. M. 1993, ApJ, 411, 153

Bottema, R., van der Kruit, P. C., \& Valentijn, E. A. 1991, A\&A, 247, 357

Caldwell, N., Rose, J. A., \& Concannon, K. D. 2003, AJ, 125, 2891

Cardiel, N. 1999, Ph.D. Thesis, Universidad Complutense de Madrid

Cardiel, N., Gorgas, J., \& Aragón-Salamanca, A. 1995, MNRAS, 277,502

Cardiel, N., Gorgas, J., Cenarro, A. J., \& González, J. J. 1998, A\&AS, 127, 597

Carlberg, R. G. 1984, ApJ, 286, 403

Carollo, C. M., Danziger, I. J., \& Buson, L. M. 1993, MNRAS, 265, 553

Courteau, S., de Jong, R. S., \& Broeils, A. H. 1996, ApJ, 457, L73

de Jong, R. S., Simard, L., Davies, R. L., et al. 2004, MNRAS, 355, 1155

Denicoló, G., Terlevich, R., Terlevich, E., et al. 2005, MNRAS, 356, 1440

di Nella, H., García, A. M., Garnier R., \& Paturel, G. 1995, A\&AS, 113, 151

Falcón-Barroso, J., Peletier, R. F., \& Balcells, M. 2002, MNRAS, 335, 741

Falcón-Barroso, J., Peletier, R. F., Emsellem, E., et al., 2004, MNRAS, 350, 35

Fisher, D., Franx, M., \& Illingworth, G. D. 1996, ApJ, 459, 110

Friedli, D. 1998, in Abundance Profiles: Diagnostic Tools for Galaxy History, ed. D. Friedli et al. (San Francisco: ASP), 287

Friedli, D., \& Benz, W. 1995, A\&A, 310, 649

Friedli, D., Benz, W., \& Kennicutt, R. 1994, ApJ, 430, L105

Golev, V., Prugniel, Ph., Simien, F., \& Longhetti, M. 1999, A\&AS, 136, 519

González, J. J. 1993, Ph.D. Thesis, University of California, Santa Cruz

González, J. J., \& Gorgas, J. 1996, in Fresh Views on Elliptical Galaxies, ed. A. Buzzoni, A. Renzini, \& A. Serrano (San Francisco: ASP), 225

Gorgas, J., Jablonka, P., \& Goudfrooij, P. 2003, in Highlights of Spanish Astrophysics III, ed. J. Gallego, J. Zamorano, \& N. Cardiel (The Netherlands: Kluwer Academic Pub.), 99

Goudfrooij, P., \& Emsellem, E. 1996, A\&A, 306, L45

Goudfrooij, P., Gorgas, J., \& Jablonka, P. 1999, Ap\&SS, 269, 109

Hamuy, M., Suntzeff, N. B., Heathcote, S. R., et al. 1994, PASP, 106, 566

Idiart, T.P., de Freitas Pacheco, J. A., \& Costa, R. D. D. 1996, AJ, 112, 2541

Jablonka, P., Martin, P., \& Arimoto, N. 1996, AJ, 112, 1415

Jablonka, P., Gorgas, J., \& Goudfrooij, P. 2002, Ap\&SS, 281, 367

Kauffmann, G., White, S. D. M., \& Guiderdoni, B. 1993, MNRAS, 264, 201

Kormendy, J., \& Kennicutt, R. C. 2004, ARA\&A, 42, 603

Kuntschner, H., Emsellem, E., Bacon, R., et al. 2006, MNRAS, 369, 497

Lauberts, A. 1982, The ESO/Uppsala survey of the ESO(B) atlas

Lütticke, R., Dettmar, R.-J., \& Pohlen, M. 2000, A\&AS, 145, 405

Martin, P., \& Roy, J.-R., 1994, ApJ, 426, 601

Massey, P., Strobel, K., Barnes, J. V., \& Anderson, E. 1988, ApJ, 328, 315

McArthur, L. A., Courteau, S., \& Holtzman, J. A. 2003, ApJ, 582, 689

Mehlert, D., Thomas, D., Saglia, R. P., Bender, R., \& Wegner, G. 2003, A\&A, 407, 423

Moorthy, B. K., \& Holtzman, J. A. 2006, MNRAS, 371, 583

Nilson, P. 1973, Uppsala general catalogue of galaxies, Nova Acta Regiae Societatis Scientiarum Upsaliensis, Ser. V, A, Vol. 1

Norman, C. A., Sellwood, J. A., \& Hasan, H. 1996, ApJ, 462, 114

Oke, J. B. 1990, AJ, 99, 1621

Peletier, R. F., Balcells, M., Davies, R. L., et al. 1999, MNRAS, 310, 703

Pfenniger, D., \& Norman, C. A. 1990, ApJ, 363, 391

Prugniel, Ph., Maubon, G., \& Simien, F. 2001, A\&A, 366, 68

Sánchez-Blázquez, P. 2004, Ph. D. Thesis, Universidad Complutense de Madrid

Sánchez-Blázquez, P., Gorgas, J., \& Cardiel, N. 2006a, A\&A, 457, 823

Sánchez-Blázquez, P., Gorgas, J., Cardiel, N., \& González, J. J., 2006b, A\&A, 457, 787

Sargent, W. L. W., Schechter, P. L., Boksenberg, A., \& Shortridge, K. 1977, ApJ, 212,326

Sil'chenko, O. K. 2006, ApJ, 641, 229

Trager, S. C., Worthey, G., Faber, S. M., Burstein, D., \& González, J. J. 1998, ApJS, 116, 1

Trager, S. C., Faber, S. M., Worthey, G., \& González, J. J. 2000, AJ, 119, 1645

Vila-Costas, M. B., \& Edmunds, M. G., 1992, MNRAS, 259, 121

Worthey, G., \& Ottaviani, D. L. 1997, ApJS, 111, 377

Worthey, G., Faber, S. M., González, J. J., \& Burstein, D. 1994, ApJS, 94, 687

Zaritsky, D., Kennicutt, R. C., \& Huchra, J. P. 1994, ApJ, 420, 87 